S131 AMBULATORY MANAGEMENT OF SECONDARY SPONTANEOUS PNEUMOTHORAX

RV Reddy, F Khan, M Naeem, N Siddique, I Masih, Y Vali. Kettering General Hospital, Kettering, UK

\subsection{6/thoraxinl-2017-210983.137}

Introduction and Aim Management of spontaneous pneumothorax is predominantly inpatient based despite availability of devices which facilitate ambulatory management. At our institution most patients meeting predefined criteria have outpatient management. We aimed to assess the effectiveness of ambulatory management of SSP.

Methods Data on all secondary spontaneous pneumothorax patients presenting to the emergency department between September 2014 and June 2017 was prospectively recorded. Patients were initially managed by the emergency department practitioners, usually with insertion of a Rocket seldinger (size 12 F) chest drain. They were then referred to the respiratory team at the earliest opportunity. Patients meeting eligibility criteria (age 16-80, WHO performance status $0-1$ and no coexisting condition requiring admission) had their underwater seal replaced with a Pneumostat valve (Atrium Medical Corporation) which was connected to their chest drain. They were then discharged from hospital with reviews on alternate days on the ambulatory care unit. Chest drains were removed once air leakage had stopped for 24 hours. Suction was not employed. Patients with a persistent air leak were referred to the thoracic surgeons on day five and were admitted electively from home for surgery with chest drains in-situ.

\begin{tabular}{lll} 
Abstract S131 Table 1 & & \\
\hline & $\begin{array}{l}\text { Ambulatory } \\
\text { Cohort }\end{array}$ & $\begin{array}{l}\text { Non-ambulatory } \\
\text { Cohort }\end{array}$ \\
\hline Total number of patients & 49 & 50 \\
No. requiring Chest drainage & 44 & 47 \\
Mean Age & 60 (21-76) & 70 (30-92) \\
Sex (M:F) & $26: 23$ & $38: 12$ \\
Size of pneumothorax & $40: 9$ & $32: 18$ \\
(Large:Small) & & \\
No. resolved by day 5 & $29 / 49$ (59\%) & $32 / 50$ (64\%) \\
No. requiring surgery due to non- & 9 & 4 \\
resolution & & \\
Mean length of drainage (days) & 5.57 & 7.96 \\
$\begin{array}{l}\text { Mean length of out-patient (OP) } \\
\text { drainage }\end{array}$ & 4.77 (out of 5.57) & NA \\
Mean Number of OP reviews & 2.08 & \\
Total number of complications & 6 & NA \\
$\begin{array}{l}\text { Mean pain score (scale 0-10) } \\
\text { Mean Satisfaction score (scale 0-5) }\end{array}$ & 3.65 & 15 \\
\hline & 4.95 & NA \\
\hline
\end{tabular}

Results Data on all 99 consecutive patients with spontaneous pneumothorax were collected prospectively. Patient characteristics and outcomes are shown in Table 1. 55 episodes qualified for ambulatory management of which 49 SSP had outpatient management. The six patients who were not treated on the ambulatory pathway had resolution of pneumothorax by day 2. Of the 49 SSP who had ambulatory treatment, nine $(18.37 \%)$ required surgery due to non-resolution whilst 11 achieved resolution between 6 and 19 days. There were a total of six complications during ambulatory management.
Three patients experienced drain blockage which necessitated replacement of the tube. Two patients developed empyema; one of these was following prolonged drainage (19 days) as he declined surgery. One patient's drain fell out but did not require reinsertion as the pneumothorax had already healed. Conclusion This study confirms that the use of chest drains with one-way valves in the ambulatory management of selected secondary spontaneous pneumothoraces is safe with few complications.

\section{S132 CONSERVATIVE MANAGEMENT IN TRAUMATIC PNEUMOTHORACES: AN OBSERVATIONAL STUDY}

${ }^{1} \mathrm{~S}$ Walker, ${ }^{2} \mathrm{~S}$ Barratt, ${ }^{2} \mathrm{~J}$ Thompson, ${ }^{1} \mathrm{~N}$ Maskell. 'Academic Respiratory Unit, Bristol, UK; ${ }^{2}$ Southmead Hospital, Bristol, UK

\subsection{6/thoraxjnl-2017-210983.138}

Background Traumatic pneumothoraces are a common consequence of major trauma. Despite this, there is a paucity of literature regarding their optimal management, including the role of conservative treatment. The aim of this study was to assess the treatment, complications and outcomes of traumatic pneumothoraces in patients presenting to a Major Trauma Centre.

Methods The prospectively collected Trauma Audit :

and Research Network (TARN) database was used to identify all patients presenting with traumatic pneumothoraces to a UK Major Trauma Centre from April 2012 to December 2016. Demographics, mechanism of injury, injury severity score (ISS), management and outcomes were analysed.

Results 602 patients were included in study period. Mean age 48 (SD 22) with 73\% male. Mean ISS was 26 and inpatient mortality 9\%. Of the 602 traumatic pneumothoraces, 277/602 $(46 \%)$ were initially treated conservatively. 252/277 (90\%) of this cohort did not require subsequent chest tube insertion, including the majority, 56/62 (90\%), of patients on admission positive pressure ventilation (PPV). Hazard ratio for failure of conservative management showed no difference between the ventilated and non-ventilated patients (HR $1.1 \mathrm{p}$ 0.84). Only the presence of large hemothorax was associated with increased likelihood of failure of conservative management.

Conclusions In the largest observational study of traumatic pneumothoraces published to date, over $90 \%$ of patients whose pneumothorax was managed conservatively never required subsequent tube drainage. Importantly, this also applies to patients requiring PPV, with no significant increased risk of failure of expectant management. This data supports a role for conservative management in traumatic pneumothoraces.

\section{Core outcomes for mechanical ventilation}

\section{S133 A CORE OUTCOME SET FOR MECHANICAL VENTILATION TRIALS: THE COVENT STUDY}

${ }^{1} \mathrm{SM}$ Ringrow, ${ }^{1} \mathrm{DF}$ MCAuley, ${ }^{1} \mathrm{M}$ Clarke, ${ }^{2} \mathrm{JC}$ Marshall, ${ }^{3} \mathrm{~B}$ Connolly, ${ }^{4} \mathrm{~L}$ Rose, ${ }^{1} \mathrm{~B}$ Blackwood. 'Queens University Belfast, Belfast, UK; ${ }^{2}$ St Michael's Hospital, Toronto, Canada; ${ }^{3}$ Lane Fox Clinical Respiratory Research Centre, London, UK; ${ }^{4}$ Sunnybrook Health Sciences Centre, Toronto, Canada

10.1136/thoraxjnl-2017-210983.139 
There is inconsistency in the selection and measurement of outcomes in clinical trials of mechanically ventilated critically ill patients. ${ }^{1}$ This presents challenges when comparing trials, and particularly when undertaking meta-analysis of trial data. A core outcome set (COS) is a minimum set of standardised outcomes that should be reported in every trial of a specific intervention. We therefore aimed to develop a COS for use in future trials where the aim of the intervention is to modify the duration of mechanical ventilation. Mixed consensus methods were used to develop this COS. A large, international, online Delphi study was followed by 2 consensus webinars with representatives from the Delphi panel and additional input from a separate patient representative group teleconference. Participants were recruited via international trials groups, critical care societies, charities and associations. Additional researchers were identified through a PubMed search. Participants represented 4 main stakeholder groups; patients, clinicians, researchers and industry. The study was conducted between December 2015 and October 2016. The Delphi ran over 3 rounds; Round 1 included 24 outcomes obtained from a systematic review. During this round a further 23 outcomes were proposed and added by participants. Numbers of participants completing each round were 200, 178 and 161 respectively. A total of 19 outcomes gained consensus through the Delphi process and were discussed at the consensus webinars and the patient teleconference. The outcomes in the final COS were agreed across all 3 meetings and included mortality, health-related quality of life, duration of mechanical ventilation, reintubation, length of stay and successful extubation (Table 1). Using robust consensus methodology this COS has been developed for use in all trials and systematic reviews evaluating interventions that may reduce the duration of mechanical ventilation. Agreement now needs to be reached on how these outcomes should be measured and defined.

\section{REFERENCE}

1. Blackwood B, Clarke M, McAuley DF, McGuigan PJ, Marshall JC, Rose L. How outcomes are defined in clinical trials of mechanically ventilated adults and children. American Journal of Respiratory and Critical Care Medicine 2014, Apr 15;189(8):886.

\begin{tabular}{llll}
$\begin{array}{l}\text { Abstract S133 Table } 1 \text { Core outcomes agreed at each consensus } \\
\text { meeting and the final COS }\end{array}$ & & \\
\hline Webinar 1 & Webinar2 & Teleconference & Final COS \\
\hline Mortality & Mortality & Mortality & Mortality \\
HRQOL & HRQOL & HRQOL & HRQOL \\
Duration IMV & Duration IMV & Duration IMV & Duration IMV \\
Reintubation & Reintubation & Reintubation & Reintubation \\
Length of Stay & Length of Stay & Length of Stay & Length of \\
& & & Stay \\
Successful Extubation & Successful & Successful & Successful \\
& Extubation & Extubation & Extubation \\
& & Delirium & \\
& Survival & Survival & \\
Pulmonary & & Pulmonary & \\
Complications & & Complications &
\end{tabular}

Key: HRQOL, health related quality of life; IMV, invasive mechanical ventilation

\section{S134 \\ EFFECT OF CONTINUOUS POSITIVE AIRWAY PRESSURE ON NEURAL RESPIRATORY DRIVE AND FUNCTIONAL CAPACITY IN EXCESSIVE DYNAMIC AIRWAY COLLAPSE PATIENTS}

${ }^{1} \mathrm{G}$ Kaltsakas, ${ }^{1} \mathrm{M}$ Patout, ${ }^{1} \mathrm{G}$ Arbane, ${ }^{2} \mathrm{~L}$ Ahmed, ${ }^{3} \mathrm{D}$ D'Cruz, ${ }^{4} \mathrm{M}$ Polkey, 5 Hull, ${ }^{1} \mathrm{~N}$ Hart, ${ }^{1}$ PB Murphy. 'Lane Fox Respiratory Unit, Guy's and St Thomas' NHS Foundation Trust, London, UK; 'Department of Thoracic Medicine, Guy's and St Thomas' NHS Foundation Trust, London, UK; ${ }^{3}$ Louise Coote Unit, Guy's and St Thomas NHS Foundation Trust, London, UK; ${ }^{4}$ NIHR Respiratory Biomedical Research Unit at the Royal Brompton and Harefield Foundation NHS Trust and Imperial College, London, UK; ${ }^{5}$ Department of Respiratory Medicine, Royal Brompton Hospital, London, UK

\subsection{6/thoraxjnl-2017-210983.140}

Excessive dynamic airway collapse (EDAC) and tracheobronchomalacia (TBM) occur due to weakening of the walls of the central airways leading to airway collapse on expiration. Positive airway pressure provides a pneumatic stent maintaining airway patency. CPAP is used to prevent airway collapse during sleep, but could also facilitate improved exercise capacity in this patient group. The aim of this study was to investigate the effect of ambulatory continuous positive airway pressure (CPAP) on neural respiratory drive and exercise capacity. Patients with CT or bronchoscopic evidence of EDAC or TBM underwent baseline testing and $6 \mathrm{~min}$ walk test (6MWT). Physiological testing was performed with patients self-ventilating and on CPAP at 4,7 and $10 \mathrm{~cm} \mathrm{H}_{2} \mathrm{O}$ to identify optimal ambulatory CPAP pressure. Patients then underwent repeat 6MWT on sham or active CPAP in a random order. Neural respiratory drive index (NRDI) was assessed by surface electromyography of the parasternal intercostals (EMGpara \%max $\chi$ respiratory rate) while self-ventilating and on

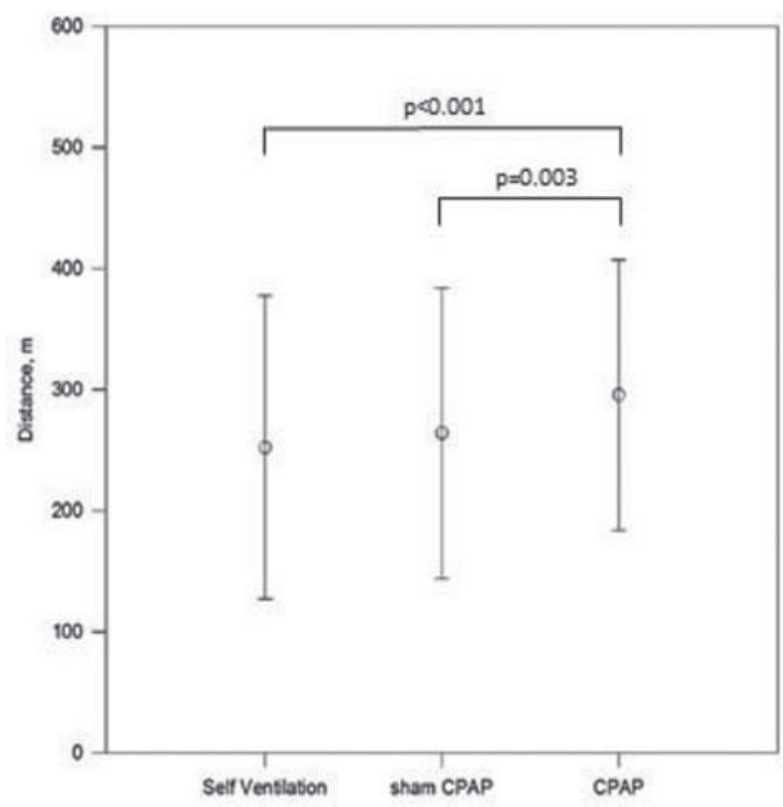

Abstract S134 Figure 1 The 6MWT while on optimal CPAP was increased comparing to self-ventilation and sham CPAP. 